\title{
A SYSTEMATIC CHANGE WITH TIME IN THE SIZE OF BETELGEUSE
}

\author{
C. H. Townes ${ }^{1}$, E. H. Wishnow ${ }^{1}$, D. D. S. Hale ${ }^{2}$, And B. WalP ${ }^{1}$ \\ ${ }^{1}$ Space Sciences Laboratory and Department of Physics, University of California, Berkeley, CA 94720, USA; cht@ ssl.berkeley.edu, wishnow@ssl.berkeley.edu, \\ bernie@isi.mtwilson.edu \\ ${ }^{2}$ Department of Astronomy, California Institute of Technology, Pasadena, CA 91125, USA; dhale @astro.caltech.edu \\ Received 2009 March 9; accepted 2009 April 20; published 2009 May 11
}

\begin{abstract}
The diameter of Betelgeuse ( $\alpha$ Orionis) has been measured at a wavelength of $11.15 \mu \mathrm{m}$ using the Infrared Spatial Interferometer over the past 15 years. During this 1993-2009 time period the star's size has decreased systematically by $15 \%$.
\end{abstract}

Key words: stars: general - stars: individual (Betelgeuse) - techniques: interferometric

\section{INTRODUCTION}

Betelgeuse ( $\alpha$ Orionis) was the first star to be measured in size. Michelson \& Pease (1921) reported a diameter of 0.047 at optical wavelengths, but in addition to a measurement uncertainty of $10 \%$, they estimated that its actual size was probably 10\%-15\% larger because of limb darkening. Since then it has been measured from time to time by a number of interferometers at various wavelengths, giving values that vary substantially with wavelength. It has now been measured systematically over a period of about 15 years with the Infrared Spatial Interferometer (ISI). The ISI uses a narrow frequency range to avoid spectral lines and mid-infrared (mid-IR) wavelengths which penetrate the surrounding dust well. The diameters measured by the ISI are not particularly inconsistent with previous measurements, are rather accurate, and show clearly that the star has systematically decreased in size by about $15 \%$ over the past 15 years.

\section{GENERAL DISCUSSION}

Since all of the interferometric measurements made, except those of the ISI, have used rather broad bandwidths it is not surprising that there have been large variations in the resulting apparent size. What most interferometers measure is partly emission from spectral lines of gas surrounding the star and partly absorption by spectral lines, as well as effects of dust around the star, rather than the stellar continuum directly. Variations in apparent size with wavelength have been previously tabulated (Weiner et al. 2003; Perrin et al. 2004). The variations in apparent diameters from visible wavelengths to $3 \mu \mathrm{m}$ are as large as about $50 \%$.

The ISI operates at wavelengths near $11 \mu \mathrm{m}$, using heterodyne detection with $\mathrm{CO}_{2}$ lasers as local oscillators. This converts the infrared signal into a microwave frequency signal with a bandwidth of about $5.4 \times 10^{9} \mathrm{~Hz}$, or $0.18 \mathrm{~cm}^{-1}$. The system is tunable so that wavelengths can be chosen to avoid any spectral lines. Measurements reported here were thus made in a narrow frequency range which is free of spectral lines, importantly including any substantial ones due to water vapor. The radiation from the surrounding dust is easily detected, but at 11 $\mu \mathrm{m}$ wavelength the dust is also rather well penetrated. The distribution of dust and the stellar characteristics are hence easily and clearly separated. Since this wavelength is not scattered as much as shorter ones, the ISI views the stellar continuum rather clearly and directly and gives a reliable measure of the real size of the star, as defined by its effective continuum diameter. Inter-

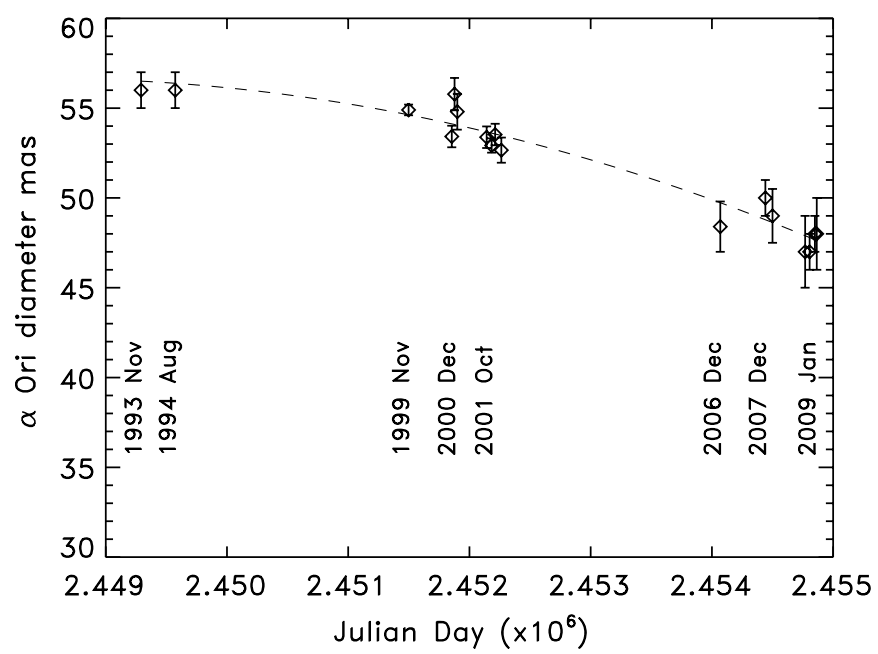

Figure 1. Diameter of $\alpha$ Ori measured at $11.15 \mu \mathrm{m}$ by the ISI over 15 years. Values previously published are: 1994, Bester et al. (1996); 1999, 2000, and 2001, Weiner et al. (2003); 2006, Tatebe et al. (2007). Values from 2007 to 2009 are new measurements. The dashed line is a quadratic fit to the data.

ferometric measurements with the ISI began in 1993 with two movable telescopes that can provide a wide variety of baselines. A third telescope was added in 2002 so that stellar diameters could be measured simultaneously from three directions and point inversion asymmetries detected by phase closure, using the phases of three simultaneous interference signals.

In 2006, the ISI detected a major hot spot on $\alpha$ Ori, producing about $10 \%$ of the total flux at $11.15 \mu \mathrm{m}$, and resulting in some asymmetry in the radiation intensity (Tatebe et al. 2007). This discovery is consistent with theoretical predictions by Schwarzschild (1975). Without correcting for a hot spot, the apparent diameters measured by individual differently oriented baselines differed substantially (Tatebe et al. 2007). But modeling the system as a uniform disk plus a hot spot on one side of the star gives an effective circular diameter for the star of $48.4 \pm 1.4$ mas. Other ISI measurements, between 1994 and 2009, did not indicate any large hot spots or asymmetries, though these would not have been detected by the ISI before the third telescope was added in 2002.

\section{RESULTS AND CONCLUSIONS}

Measurements of $\alpha$ Ori's diameter over the period 1994 2009 show a substantial and systematic change over this period, 
Table 1

ISI Diameter Measurements of Betelgeuse 1993-2009

\begin{tabular}{lcc}
\hline \hline Date (average) & Diameter (mas) & Reference \\
\hline 1993 Oct 30 & $56.0 \pm 1.0$ & 1 \\
1994 Aug 8 & $56.0 \pm 1.0$ & 1 \\
1999 Nov 15 & $54.9 \pm 0.3$ & 2 \\
2000 Nov 5 & $53.4 \pm 0.6$ & 2 \\
2000 Nov 28 & $55.8 \pm 0.9$ & 2 \\
2000 Dec 21 & $54.8 \pm 1.0$ & 2 \\
2001 Aug 20 & $53.4 \pm 0.6$ & 2 \\
2001 Sep 30 & $52.9 \pm 0.4$ & 2 \\
2001 Oct 29 & $53.5 \pm 0.6$ & 2 \\
2001 Dec 19 & $52.7 \pm 0.7$ & 2 \\
2006 Nov 29 & $48.4 \pm 1.4$ & 3 \\
2007 Dec 6 & $50.0 \pm 1.0$ & \\
2008 Feb 3 & $49.0 \pm 1.5$ & \\
2008 Oct 29 & $47.0 \pm 2.0$ & \\
2008 Dec 5 & $47.0 \pm 1.0$ & \\
2009 Jan 17 & $48.0 \pm 1.0$ & \\
2009 Feb 2 & $48.0 \pm 2.0$ & \\
\hline
\end{tabular}

Reference. (1) Bester et al. 1996; (2) Weiner et al. 2003; (3) Tatebe et al. 2007.

as may be seen in Figure 1 and Table 1. Data prior to 2007 have been previously reported and the data since this time are new. The measurements were made with baselines of $32-59 \mathrm{~m}$, which allowed adequate resolution of the star. ISI measurements with shorter baselines, sensitive to spatial frequencies related to the dust shells, are not included in the figure. Variations in the luminosity and diameters measured for $\alpha$ Ori have been previously reported by White (1980), but the data are not good enough to provide very clear-cut conclusions. Also, the modest known variations in luminosity are probably not simply related to size and the visible band photometric record of the AAVSO does not resemble the size variation of Figure 1. Some of the complexities of radiation from $\alpha$ Ori, particularly due to surrounding molecular gas, are illustrated in the recent report of ultraviolet, IR, and mm studies of the star by Harper et al. (2009).

ISI measurements over the last 15 years clearly show a systematic change in the diameter of $\alpha$ Ori. This change may or may not be periodic; if it is, the period is likely rather long, perhaps a few decades. One might attribute some of the change in apparent size to a specific instability connected with the large spot in 2006, but this would only be a possible conjecture. It should be valuable to continue accurate measurements of $\alpha$ Ori's size and other characteristics in order to understand the dynamics involved in this striking change, and to have systematic long-term measurements of similar stars.

We thank W. Fitelson for his efforts in developing and keeping the ISI operating during these measurements. We also thank M. Bester, W. C. Danchi, and P. G. Tuthill, former staff members, the former graduate students, E. A. Lipman, J. D. Monnier, J. Wiener, and K. Tatebe, and the many undergraduate students who have helped perform and analyze these measurements. We acknowledge the use of the AAVSO database. This work received support from the National Science Foundation, the Office of Naval Research, and the Gordon and Betty Moore Foundation.

Facility: ISI

\section{REFERENCES}

Bester, M., Danchi, W. C., Hale, D. D. S., Townes, C. H., Degiacomi, C. E., Mekarnia, D., \& Geballe, T. R. 1996, ApJ, 463, 336

Harper, G. M., Carpenter, K. G., Ryde, N., Smith, N., Brown, J., Brown, A., \& Hinkle, K. H. 2009, Am. Inst. Phys. Conf. Ser., 1094, 868

Michelson, A. A., \& Pease, F. G. 1921, PNAS, 7, 143

Perrin, G., Ridgeway, S. T., Coudé du Foresto, V., Mennesson, B., Traub, W. A., \& Lacasse, M. G. 2004, A\&A, 418, 675

Schwarzschild, M. 1975, ApJ, 195, 137

Tatebe, K., Chandler, A. A., Wishnow, E. H., Hale, D. D. S., \& Townes, C. H. 2007, ApJ, 670, L21

Weiner, J., Hale, D. D. S., \& Townes, C. H. 2003, ApJ, 589, 976

White, N. M. 1980, ApJ, 242, 646 